\title{
Matrix Analysis of Repetitive Circulant Structures: New-block and Near Block Matrices
}

\author{
Ali Kaveh ${ }^{1 *}$, Ali Joudaki ${ }^{1}$ \\ ${ }^{1}$ Centre of Excellence for Fundamental Studies in Structural Engineering, \\ School of Civil Engineering, \\ Iran University of Science and Technology, \\ Narmak, Tehran-16, Iran \\ ${ }^{*}$ Corresponding author, e-mail: alikaveh@iust.ac.ir
}

Received: 10 April 2019, Accepted: 30 April 2019, Published online: 28 May 2019

\begin{abstract}
In many scientific fields and several problems, Block Circulant Matrices (BCM) have been used for a long period of time. Each row of the BCM is a cyclic shift of its upper row to the right. BCM has been studied widely and there are closed-form solutions for problems of BCM. In these problems, the properties of near-BCM and BCM lead to a significant decrease in computational cost and efforts. In other words, these matrices are useful to perform some computational operations at the low cost. This study introduces a method for transforming a structure into a new type of Block Circulant Structure (BCS) by applying minor modifications. Furthermore, transformation of structural matrices into Block Circulant Matrices is discussed, and the properties of these matrices are then described in details. The methods introduce calculating eigenvalues and eigenvectors of these matrices instead of calculating the inverse of their matrices. To achieve this goal, the properties of near-Block and Block Circulant Matrices are used to analyze the structural stiffness matrices. In addition, the inverse of stiffness matrices for structures are calculated and utilized in structural mechanics. For clarification of efficiency and accuracy of the method, some examples are presented.
\end{abstract}

Keywords

block circulant matrices, near block circulant matrices, transformation, eigenvalues

\section{Introduction}

There are many mathematical concepts that can simplify problems and reduce computational cost while preserving the accuracy of the solutions. Block Circulant Matrix (BCM) has been already applied to many problems, moreover the concepts of BCM have helped structural mechanics for solving some of its problems. Mathematical rules, linear algebra and graph theory have some techniques by which calculation cost and size of the structural matrices can be reduced in repetitive, regular and circulant structures. If a structural model can be considered as the product of two or three subgraphs, it is called regular and if all collections of identical parts are connected together in a regular form, it is called a repetitive structure. Symmetry and regularity of structures are discussed in details in books by Kaveh [1] and [2]. Repetitive structures have been widely studied by Kangwai et al. [3], Kangwai and Guest [4], and Zingoni [5] and [6].
In this paper, some results of researches which are performed by Kaveh and Rahami [7, 8] are extended. They simplified the model of regular structures by using graph products and linear algebra. Also they presented various types of circulant structures. Some irregular structures were analyzed by applying modifications [9, 10]. Static, modal analysis and free vibration of structures with some repetitive patterns have been studied [11], also some structures with different patterns are introduced. As results, some methods for nodal numbering and a method for analyzing these structures are presented. Shojaei et al. [12] performed static and dynamic analysis of structures using graph product rules. In this study, Block Circulant Structure (BCS) and near-Block Circulant Structure were investigated in the form of new-Block Circulant Structure (BCS) with some modifications. It is noticeable that, Block Circulant Structure (BCS) and new-Block Circulant Structure (BCS) have different properties. 
Kaveh et al. [13] investigated the symmetric regular graphs and developed a method for symmetry analysis of regular structures. They computed the eigensolution of some special graphs using a combined graph-group method. They employed graph products to improve the conventional group theoretic method for decomposition of symmetric graphs. Kaveh et al. [14] used graph products to analyze the natural frequencies of the cable networks. In this method, they decomposed the structure matrix into submatrices of low dimensions. In some cases, these matrices are completely diagonal and have obtained their natural frequencies.

Kaveh and Fazli [15] performed structural systems analysis that had partial perturbations. In this method, they modified the formulation for the eigenproblem of these structures and obtained few lower eigenpairs of large-scale modified regular structures. The proposed method uses regular base model because it is simpler to analyze. Kaveh and Fazli [16] studied symmetric/regular structural configurations that can be shown by canonical forms. They also used a numerical method to extend the calculating concepts of matrix canonical forms to nonconforming regular structures. Kaveh and Fazli [17] proposed a numerical method based on single vector iterations to find several eigenpairs of modified system. In this method they used the characteristics and information derived from a decomposable model called the base model.

Kaveh et al. [18] presented a combined force method for analysis of near regular structures using the results of regular structures. These decompositions lead to the preferential solution of structures by converting the main problem into less complicated subproblems. Shojaei et al. [19] Examined finite element models of regular and near-regular systems using the rules of graph products. In finite element models, due to element repetition, regular/near regular patterns are very common in these models and regularity in their stiffness matrix is very common. They used the Kronecker products and the canonical forms to simplify the finite element analysis of planar elements. Kaveh and Shojaei [20] studied some of the different methods for efficient and optimal analysis of structures. They investigated the regular structures with extra nodes or elements. They obtained fast and optimal solution for design process by manipulation of the stiffness matrix and by using iterative methods.

In this paper, special structures named near-BCS and near-new-BCS are investigated. First, basic concepts and the required preliminary theories are presented, and it is shown that such matrices can be written as a sum of the Kronecker products of several matrix blocks. Also, eigenpairs of these matrices can be computed readily, and using the properties of the Kronecker products, their inverse matrices are calculated. Transformation of near-BCS and near-new-BC to BCS and new-BCS, respectively, are discussed later in this paper. The inversion of stiffness matrix can be carried out using the properties of BCM and new$\mathrm{BCM}$. The aim of this article is to show that near-newBCS or near-BCS can be decomposed into the sum of two matrices which are a low-rank matrix and a BCM or new $\mathrm{BCM}$, and then these matrices are solved the proposed method. Numerical examples are also given to support the theoretical results.

In Section 1, the difference between block and newBlock Circulant Structure (BCS) is described, and a method to convert regular structures into Block Circulant Structure (BCS) is presented and in which cylindrical coordinate system must be used. In Sections 2 and 3, Block Circulant Matrix (BCM) and near-Block Circulant Matrix are investigated, respectively. Some properties of two types of matrices are explained and a way of using mathematical concepts for obtaining eigenpairs of $\mathrm{BCM}$ and new-BCM are shown. In Sections 4 and 5, near-block and near-new-Block Circulant Structure are introduced. Firstly, some theoretical concepts are proposed so as to convert some structures to new-block or Block Circulant Structure (BCS). Then the presented method is used for considering the effects of the added parts on a structure which are analyzed. In the following, some examples are provided in which the effectiveness of the proposed method for matrix of near-BCS is illustrated. The first one is a near-BCS that needs to be reformed to become a BCS, and the second one is a plate with a rectangular hole [21] and [22], that needs to be modified to become a near-newBCS, so mathematical rules and linear algebra can be used to improve the finite element analysis. In Section 6, the conclusion is presented.

\section{Block Circulant Structure and new-Block Circulant Structure}

There are many Block Circulant Structures (BCS) such as cooling towers, domes, etc. Such structures have mathematical properties that can be more beneficial for reduction in cost of their analysis.

The general form of a Block Circulant Matrix in cylindrical coordinate can be expressed as: 


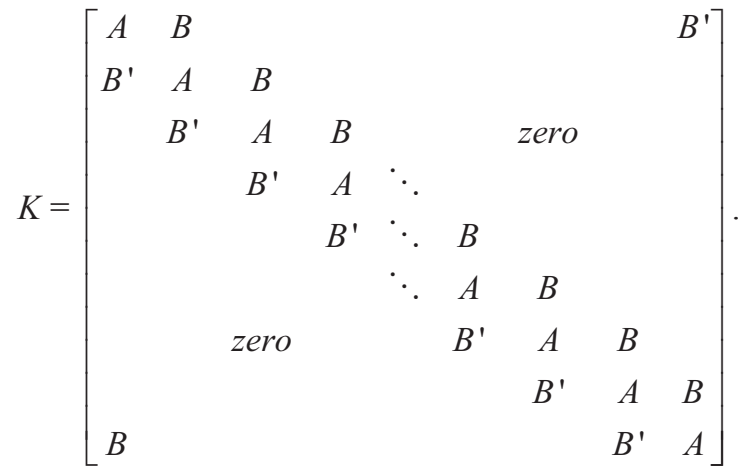

This matrix is an arrangement of blocks A, B, and B'. Any row of the Block Circulant Matrices is a cyclic shift of the upper row to the right. There are two blocks B and $\mathrm{B}^{\prime}$ in the corners of the matrix, indicating that the nodes of the beginning and the end of the structure are connected together. Furthermore, there are structures that can be modified in the form of a Block Circulant Matrix by changing in their matrices. For instance, consider the following matrix which, unlike BCM, is obtained using Cartesian coordinates:

In the following matrix Cartesian coordinate system is utilized to calculate stiffness matrix instead of Cylindrical coordinate system which is used in BCM.

$$
K=\left[\begin{array}{cccccccccc}
C & D & & & & & & & \\
D^{\prime} & C & D & & & & & & \\
& D^{\prime} & C & D & & & \text { zero } & & \\
& & D^{\prime} & C & \ddots & & & & \\
& & & D^{\prime} & \ddots & D & & & \\
& & & & \ddots & C & D & & \\
& & \text { zero } & & & D^{\prime} & C & D & \\
& & & & & & D^{\prime} & C & D \\
& & & & & & & D^{\prime} & C
\end{array}\right] .
$$

$$
K=\left[\begin{array}{cccccccccc}
A & B & & & & & & & B^{\prime} \\
B^{\prime} & A & B & & & & & & \\
& B^{\prime} & A & B & & & \text { zero } & & \\
& & B^{\prime} & A & \ddots & & & & \\
& & & B^{\prime} & \ddots & B & & & \\
& & & & \ddots & A & B & & \\
& & & & & & B^{\prime} & A & B \\
B & & & & & & & B^{\prime} & A
\end{array}\right]_{t \times t}
$$

$$
V^{\prime} K V=\sum_{i=1}^{t} P^{i} \otimes A_{i}=\left[\begin{array}{cccc}
H(1) & & & \\
& H(\omega) & & \\
& H\left(\omega^{2}\right) & \\
& & \ddots & \\
& & & H\left(\omega^{t-1}\right)
\end{array}\right]_{t \times t}
$$


Eigenvalues and eigenvectors of Block Circulant Matrix " $K$ " can be defined as:

$$
\begin{aligned}
& K=\left(\sum_{i=1}^{t} P^{i} \otimes A_{i}\right)(v \otimes u)=\sum_{i=1}^{t} P^{i} v \otimes A_{i} u=\sum_{i=1}^{t}\left(\omega^{i-1} v \otimes A_{i} u\right)= \\
& \sum_{i=1}^{t}\left(\omega^{i-1} \otimes A_{i}\right)(v \otimes u)=H\left(\omega^{i}\right)(v \otimes u) .
\end{aligned}
$$

According to Eq. (6), the eigenvalues and eigenvectors of the Block Circulant Matrix $K$ are $H(\omega)$ and $(v \otimes u)$, respectively.

\section{New-Block Circulant Matrix (new BCM)}

The new-Block Circulant Matrix has minus blocks in the corners and therefore the properties of Block Circulant Matrix cannot be used, and needs new formulation.

$K=\left[\begin{array}{cccccccccc}C & D & & & & & & & -D^{\prime} \\ D^{\prime} & C & D & & & & & & \\ & D^{\prime} & C & D & & & \text { zero } & & \\ & & D^{\prime} & C & \ddots & & & & \\ & & & D^{\prime} & \ddots & D & & & \\ & & & & \ddots & C & D & & \\ & & \text { zero } & & & D^{\prime} & C & D & \\ & & & & & & D^{\prime} & C & D \\ -D & & & & & & & D^{\prime} & C\end{array}\right]_{t \times t}$

$=I \otimes C+P \otimes D+P^{\prime} \otimes D^{\prime}$.

$P=\left[\begin{array}{cccc} & 1 & & \\ & & \ddots & \\ & \text { zero } & & 1 \\ -1 & & & \end{array}\right]_{n \times m}$.

$I$ is an identity matrix of size $n$.

Eigenpairs of the new-Block Circulant Matrix is based on Eq. (9):

$$
\begin{aligned}
& \operatorname{eig}(K)=\operatorname{eig}\left(I \otimes C+P \otimes D+P^{\prime} \otimes D^{\prime}\right)= \\
& \bigcup_{i=1}^{n}\left(C+\lambda_{i} D+\bar{\lambda}_{i} D^{\prime}\right) . \\
& \lambda^{n}=-1 \rightarrow \lambda_{i}=\exp \left(\frac{(2 k-1) \pi i}{n}\right), i=1,2, \ldots, n .
\end{aligned}
$$

$$
P=\frac{1}{\sqrt{n}}\left[\begin{array}{ccccc}
1 & 1 & 1 & \cdots & 1 \\
\lambda_{1} & \lambda_{2} & \lambda_{3} & \cdots & \lambda_{n} \\
\lambda_{1}^{2} & \lambda_{2}^{2} & \lambda_{3}^{2} & \cdots & \lambda_{n}^{2} \\
\vdots & \vdots & \vdots & \cdots & \vdots \\
\lambda_{1}^{n-1} & \lambda_{2}^{n-1} & \lambda_{3}^{n-1} & \cdots & \lambda_{n}^{n-1}
\end{array}\right]_{n \times n}
$$

The eigenvalues of the matrices $P$ and $P^{\prime}$ are conjugate and can be labelled as $\lambda$ and $\bar{\lambda}$, respectively. Also their eigenvectors are equal and the corresponding names are called $v$.

$V=v \otimes u$.

$K V=K(v \otimes u)=\left(I \otimes C+P \otimes D+P^{\prime} \otimes D^{\prime}\right)(v \otimes u)$

$=v \otimes\left(C+P \otimes D+P^{\prime} \otimes D^{\prime}\right) u$.

If eigenvalues and eigenvectors of

$\left(C+P \otimes D+P^{\prime} \otimes D^{\prime}\right) u$ are $\gamma$ and $u$, respectively, then:

$\left(C+P \otimes D+P^{\prime} \otimes D^{\prime}\right) u=\gamma u$.

Therefore:

$K V=\gamma(v \otimes u)$.

The eigenvalues and eigenvectors of the matrix $K$ are $\gamma$ and $(v \otimes u)$, respectively. Therefore, instead of computing of a high-order matrix, it is only necessary to calculate the eigenpair of its blocks, and the solution is generalized for the entire structure. In the following it is attempted to conduct an analysis of near-Block Circulant Structures using the BCM method.

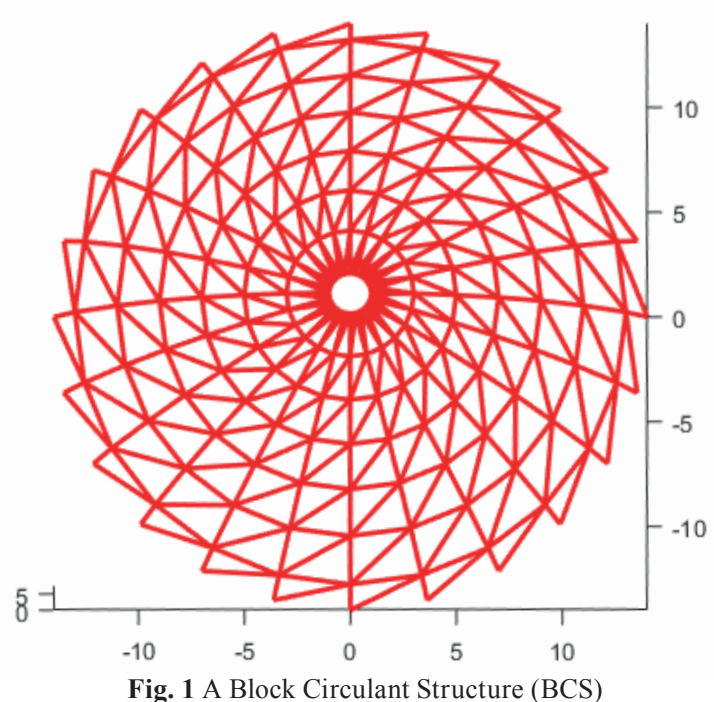




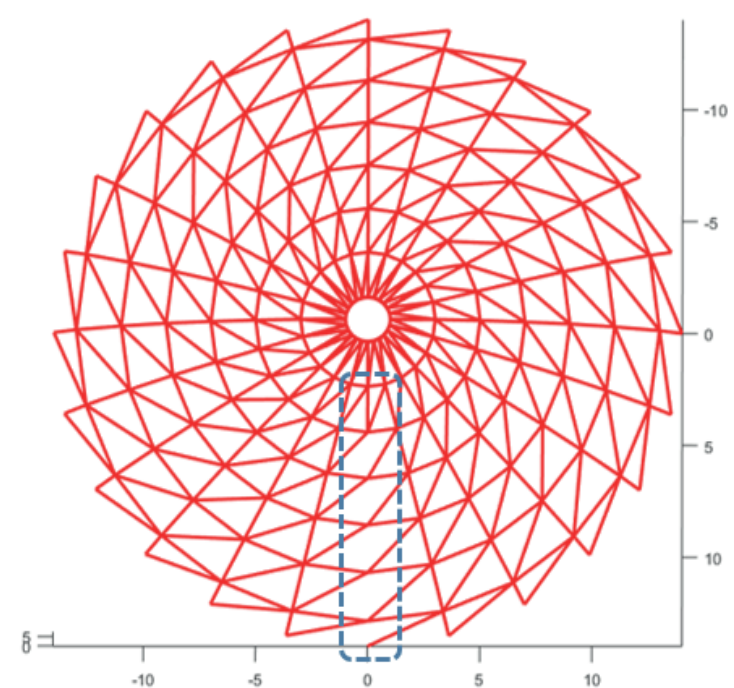

Fig. 2 A near-Block Circulant Structure (near-BCS)

\section{Analysis of near-block circulant structure using the concept of BCM}

In this section two examples are presented for the analysis of near-block circulant structures. For this purpose, the concept of block circulant matrix in utilized leading to reduction in the dimensions of the matrices to be inversed.

Example 1: Figs. 1 and 2 are Block Circulant Structure and near-Block Circulant Structure, correspondingly. In Fig. 2, some elements of Block Circulant Structure are removed and it turned into an irregular structure.

The following overall stiffness matrix of near-Block Circulant Structure (Fig. 2) in cylindrical coordinate system is presented as Eq. (16):

$K_{t}=\left[\begin{array}{ccccc}\hline a & B & \text { zero } & & B^{\prime} \\ B^{\prime} & A & B & & \\ & B^{\prime} & \ddots & \ddots & \\ & \text { zero } & \ddots & A & B \\ B & & & B & A\end{array}\right]$.

$K_{t}=K_{c}+K_{\text {added }}=\left[\begin{array}{ccccc}A & B & \text { zero } & & B^{\prime} \\ B^{\prime} & A & B & & \\ & B^{\prime} & \ddots & \ddots & \\ & \text { zero } & \ddots & A & B \\ B & & & B & A\end{array}\right]_{t \times t}$

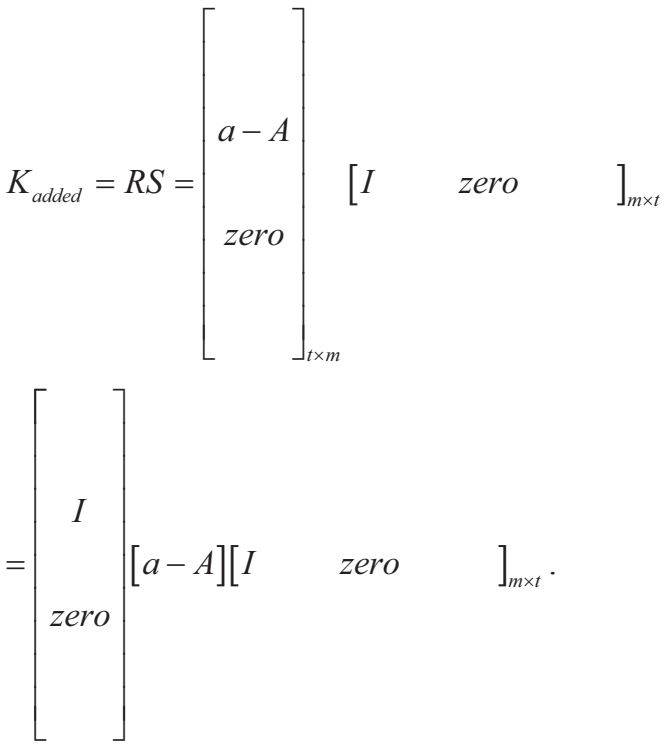

Using Eq. (19), the inverse of structural matrix of Fig. 2 can be obtained as.

$K_{t}^{-1}=\left(K_{c}+K_{\text {added }}\right)^{-1}=K_{c}^{-1}-K_{c}^{-1} R\left(I+S K_{c}^{-1} R\right)^{-1} S K_{c}^{-1}$.

The inverse of the Block Circulant Matrix can be then achieved easily. Now we want to obtain the expression $K_{c}^{-1} R\left(I+S K_{c}^{-1} R\right)^{-1} K_{c}^{-1} R$.

$$
\begin{aligned}
& I+S K_{c}^{-1} R=I_{m \times m}+S_{m \times t} K_{c t \times t}^{-1} R_{t \times m} \\
& =I_{m \times m}+K_{c \quad m \times m}^{-1}[a-A]_{m \times m} \cdot \\
& K_{c t \times t}^{-1} R_{t \times m}=K_{c t \times m}^{-1} \cdot \\
& S_{m \times t} K_{c t \times t}^{-1}=K_{c m \times t}^{-1} \cdot \\
& K_{c}^{-1} R\left(I+S K_{c}^{-1} R\right)^{-1} S K_{c}^{-1} \\
& =K_{c t \times m}^{-1}\left(I_{m \times m}+K_{c}^{-1}{ }_{m \times m}[a-A]_{m \times m}\right)^{-1} K_{c m \times t}^{-1} \cdot \\
& K_{t}^{-1}=\left(K_{c}+K_{a d d e d}\right)^{-1} \\
& =K_{c t \times t}^{-1}-K_{c t \times m}^{-1}\left(I_{m \times m}+K_{c \quad m \times m}^{-1}[a-A]_{m \times m}\right)^{-1} K_{c m \times t}^{-1} .
\end{aligned}
$$

Therefore, instead of computing the inverse of a $t \times t$ square structural matrix, it is only necessary to obtain the inverse a $t \times t$ square block circulant matrix and the inverse of an $m \times m$ square matrix. By removing the support nodes from the stiffness matrix of Fig. 1, a matrix of rank 576 is obtained. This matrix is a Block Circulant Matrix with 24 blocks of rank 24. With a slight modifications in this structure, a Block Circulant Structure (BCS) has been obtained. The order of this matrix is 576 . 
Table 1 Elapsed time of inverse matrix analysis

\begin{tabular}{lc}
\hline Method & Elapsed time (in seconds) \\
\hline $\begin{array}{l}\text { If we have reverse of the Block Circulant } \\
\text { Matrix and use the proposed method }\end{array}$ & 0.008376 \\
Direct method & 0.020217 \\
\hline
\end{tabular}

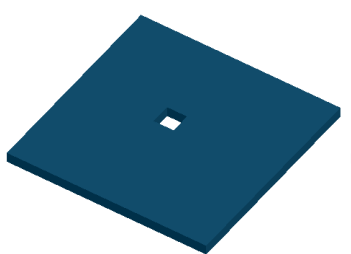

(a)

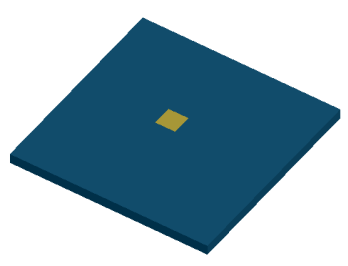

(b)

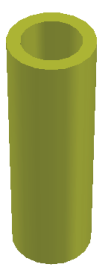

(c)
Fig. 3 (a) Unmodified plate (b) modified plate

(c) Circularized modified plate

We use the Eq. (25) to obtain the inverse of structure matrix. The results of the analysis are presented in Table 1.

$$
\begin{aligned}
& K_{t}^{-1}=\left(K_{c}+K_{\text {added }}\right)^{-1} \\
& =K_{c t \times t}^{-1}-K_{c t \times m}^{-1}\left(I_{m \times m}+K_{c m \times m}^{-1}[a-A]_{m \times m}\right)^{-1} K_{c m \times t}^{-1} .
\end{aligned}
$$

\section{Analysis of near-new-Block Circulant Structure using concept of near-BCM}

In the following example, the inverse of stiffness matrix of a plate with a square hole is calculated and analysis of the plate is presented. The global stiffness matrix is in Eq. (26).

$K_{t}=\left[\begin{array}{ccccc}a & B & \text { zero } & & \\ B^{\prime} & A & B & & \\ & B^{\prime} & \ddots & \ddots & \\ & \text { zero } & \ddots & A & B \\ & & & B & A\end{array}\right]$

$K_{t}=K_{n c}+K_{\text {added }}=\left[\begin{array}{ccccc}A & B & \text { zero } & & -B^{\prime} \\ B^{\prime} & A & B & & \\ & B^{\prime} & \ddots & \ddots & \\ & \text { zero } & \ddots & A & B \\ -B & & & B & A\end{array}\right]_{t \times t}$
$K_{\text {added }}=I_{d} * k_{\text {added }} * I_{d}^{\prime}=\left[\begin{array}{cccc}I & & & \\ & & & \\ & I & & \\ & & I & \\ & & I\end{array}\right]_{t \times 2 m}$

$\left[\begin{array}{cccc} & & & B^{\prime} \\ & a_{1}-A & b_{1}-B & \\ & b_{1}^{\prime}-B^{\prime} & a_{2}-A & \\ B & & & \end{array}\right]_{4 m \times 4 m}\left[\begin{array}{lllll}I & & & & \\ & \text { zero } & & I & \\ & & & I & \\ & & & & I\end{array}\right]_{2 m \times t}$

Using the Eqs. (27-28), inverse of the structural matrix of Fig. 3 is presented in Eq. (29).

$$
\begin{aligned}
& K_{t}^{-1}=\left(K_{c}+K_{\text {added }}\right)^{-1} \\
& =K_{c}^{-1}-K_{c}^{-1} R\left(I+S K_{c}^{-1} R\right)^{-1} S K_{c}^{-1} .
\end{aligned}
$$

The multiplication of the matrices $I_{d}$ and $I_{d}^{\prime}$ in $K_{n c}^{-1}$ causes only some of the rows and columns of the matrix $K_{n c}^{-1}$ to be entered into the computation.

Example 2: There is a square steel plate in which a hole causes asymmetry. It is also fixed along it four edges, and in the following, other parameters are mentioned:

(Size: $20 \times 20$ in, thickness: 0.1 in, modulus of elasticity: $30 \mathrm{e} 6 \mathrm{lb} / \mathrm{in}^{2}$, Poisson coefficient: 0.3 )

The plate is analyzed by using FEM in which meshing is done using elements with 4 nodes. By applying the modifications to Fig. 4(a), the corresponding matrix changes to new-Block Circulant Matrix with an extra stiffness matrix. Global stiffness matrix is achieved by deleting the support rows and columns of stiffness matrix. The global stiffness matrix is of size of 243. By making the modifications, this stiffness matrix becomes a new-Block Circulant Matrix, plus an extra stiffness matrix.

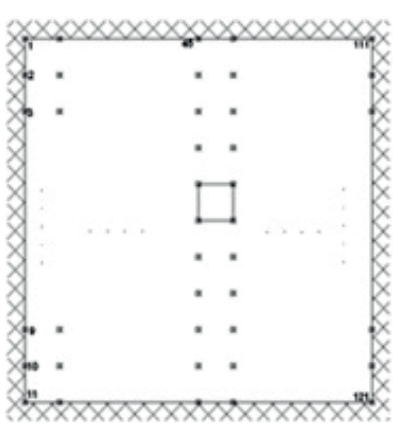

(a)

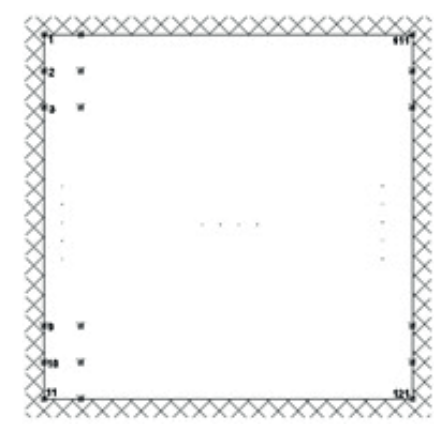

(b) (c)

Fig. 4 (a) A plate with a square hole (b) Square plate (c) Extra element 
$K_{t}^{-1}=\left(K_{n c}+K_{\text {added }}\right)^{-1}$

$=K_{n c}^{-1}-K_{n c}^{-1} * I_{d} * k_{\text {added }}\left(I_{4 m \times 4 m}+I^{\prime}{ }_{d} * K_{n c}^{-1} * I_{d} * k_{\text {added }}\right)^{-1} I_{d}^{\prime} K_{n c}^{-1}$.

$K_{\text {added }}=I_{d} * k_{\text {added }} * I_{d}^{\prime}=\left[\begin{array}{cccc}I & & & \\ & \text { zero } & & \\ & I & & \\ & & I & \\ & & & I\end{array}\right]_{t \times 2 m}$

$\left[\begin{array}{cccc} & & & B^{\prime} \\ & a_{1}-A & b_{1}-B & \\ & b_{1}^{\prime}-B^{\prime} & a_{2}-A & \\ B & & & \end{array}\right]_{4 m \times 4 m}\left[\begin{array}{lllll}I & & & & \\ & \text { zero } & & I & \\ & & & I & \\ & & & & I\end{array}\right]_{2 r}$

The reverse of Block Circulant Matrix is simply achieved. Now we want to calculate the reverse of $K_{n c}^{-1} * I_{d} * k_{\text {added }}\left(I+I_{d}^{\prime} * K_{n c}^{-1} * I_{d} * k_{\text {added }}\right)^{-1} I_{d}^{\prime} K_{n c}^{-1}$.

$I_{d}^{\prime} * K_{n c}^{-1}=\left[\begin{array}{ccc}K_{c 11}^{-1} & \cdots & K_{c 1 n}^{-1} \\ K_{c 71}^{-1} & \cdots & K_{c 7 n}^{-1} \\ K_{c 81}^{-1} & \cdots & K_{c 8 n}^{-1} \\ K_{c 91}^{-1} & \cdots & K_{c 9 n}^{-1}\end{array}\right]_{4 m \times t}$

$I_{d}^{\prime} * K_{n c}^{-1} * I_{d} * k_{\text {added }}$

$=\left[\begin{array}{llll}K_{c 11}^{-1} & K_{c 17}^{-1} & K_{c 18}^{-1} & K_{c 19}^{-1} \\ K_{c 71}^{-1} & K_{c 77}^{-1} & K_{c 78}^{-1} & K_{c 79}^{-1} \\ K_{c 81}^{-1} & K_{c 87}^{-1} & K_{c 88}^{-1} & K_{c 89}^{-1} \\ K_{c 91}^{-1} & K_{c 97}^{-1} & K_{c 98}^{-1} & K_{c 99}^{-1}\end{array}\right]_{4 m \times 4 m}$

$*\left[\begin{array}{cccc} & & & B^{\prime} \\ & a_{1}-A & b_{1}-B \\ & b_{1}^{\prime}-B^{\prime} & a_{2}-A \\ B & & \end{array}\right]_{4 m \times 4 m}$

$K_{n c}^{-1 *} I_{d}=\left[\begin{array}{rrrc}K_{c 11}^{-1} & K_{c 17}^{-1} & K_{c 18}^{-1} & K_{c 19}^{-1} \\ \vdots & \vdots & \vdots & \vdots \\ K_{c n 1}^{-1} & K_{c n 7}^{-1} & K_{c n 8}^{-1} & K_{c n 9}^{-1}\end{array}\right]_{4 m \times t}$

Therefore, instead of calculating the inverse of a $t \times t$ square matrix, it is adequate to find the inverse of a $t \times t$ square block circulant matrix and a matrix of size $4 m \times 4 m$.
If loads of 100 and $-100 \mathrm{lb}$ are applied at nodes of 51 and 21 , respectively, displacement contours will be obtained and shown in Fig. 5. The slopes in $\mathrm{x}$ and $\mathrm{y}$ directions are illustrated in Fig. 6 and Fig. 7, respectively.

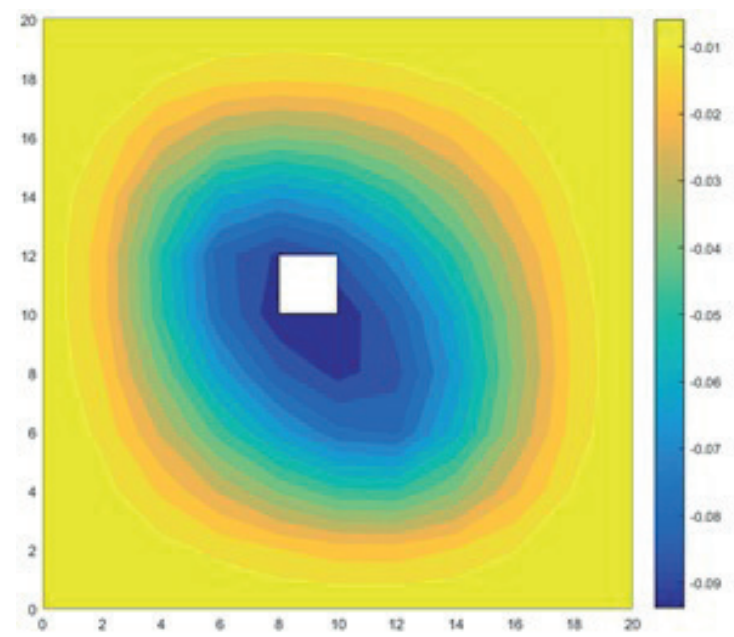

Fig. 5 Displacement contours of the plate with a square hole

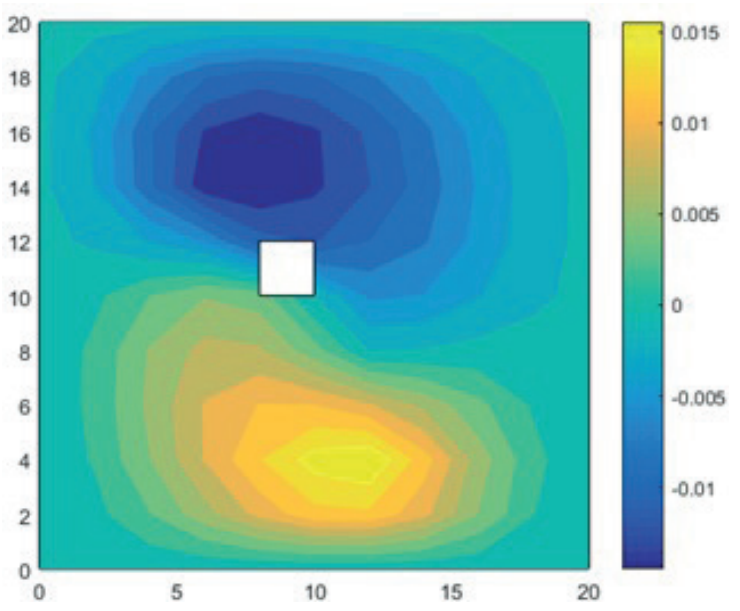

Fig. 6 Slope in $\mathrm{x}$ direction

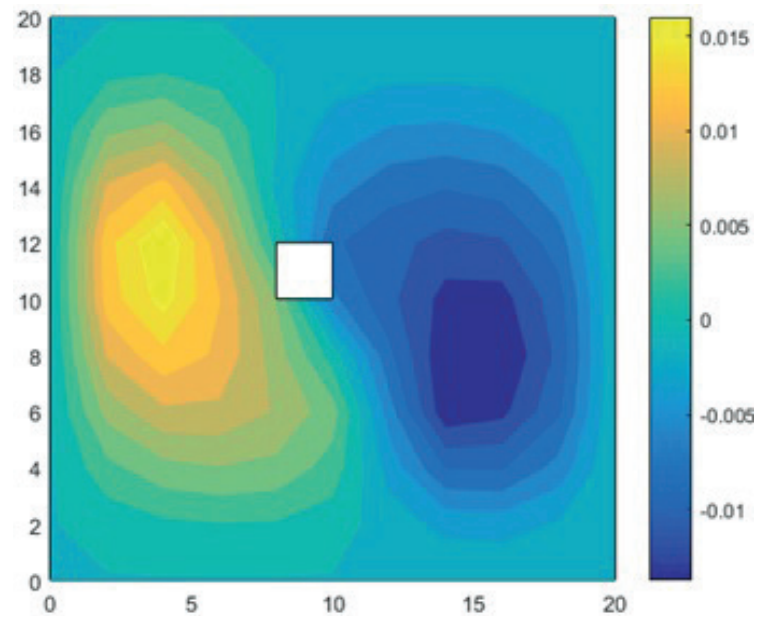

Fig. 7 Slope in y direction 


\section{Conclusions}

In this paper, we discuss block circulant and new-Block Circulant Matrices, and methods to obtain their eigenpairs. Some structures can be turned into new-Block Circulant Structure with some extra elements. A method is proposed for analysis of these structures. For Block Circulant Matrices, firstly, the structure is converted into a new-Block or Block Circulant Structure (BCS) and then, by using the proposed method, inverse of the entire matrix is obtained. In other words, if one has inverse of the Block

\section{References}

[1] Kaveh, A. "Optimal structural analysis", 2nd ed.,Wiley, Chichester, UK, 2006. https://doi.org/10.1002/9780470033326

[2] Kaveh, A. "Optimal analysis of structures by concepts of symmetry and regularity", 1st ed., Springer, Vienna, Austria, 2013. https://doi.org/10.1007/978-3-7091-1565-7

[3] Kangwai, R. D., Guest, S. D., Pellegrino, S. "An introduction to the analysis of symmetric structures", Computers and Structures, 71(6), pp. 671-688, 1999 https://doi.org/10.1016/S0045-7949(98)00234-X

[4] Kangwai, R. D., Guest, S. D. "Symmetry-adapted equilibrium matrices", International Journal of Solids and Structures, 37(11), pp. $1525-1548,2000$. https://doi.org/10.1016/S0020-7683(98)00318-7

[5] Zingoni, A. "Symmetry recognition in group-theoretic computational schemes for complex structural systems", Computers and Structures, 94-95, pp. 34-44, 2012. https://doi.org/10.1016/j.compstruc.2011.12.004

[6] Zingoni, A. "Group-theoretic insights on the vibration of symmetric structures in engineering", Philosophical Transaction of Royal Society A, 372, 2014 https://doi.org/10.1098/rsta.2012.0037

[7] Kaveh, A., Rahami, H. "Block circulant matrices and applications in free vibration analysis of cyclically repetitive structures", Acta Mechanica, 217(1-2), pp. 51-62, 2011. https://doi.org/10.1007/s00707-010-0382-x

[8] Kaveh, A., Rahami, H. "An efficient analysis of repetitive structures generated by graph products", International Journal for Numerical Methoods in Engineering, 84(1), pp. 108-126, 2010. https://oi.org/10.1002/nme.2893

[9] Kaveh, A., Rahami, H., Jodaki, A. "Analysis of repetitive and near-repetitive structures by transformation to equivalent circulant structures", Engineering Computations, 34(2), pp. 343-363, 2017. https://doi.org/10.1108/EC-01-2016-0032

[10] Kaveh, A., Pishghadam, M., Jafarvand, A. "Frequencies of Some Near-Regular Structures: A Combined Graph Product and Bisection Method", Iranian Journal of Science and Technology, Transactions of Civil Engineering, 2018. https://doi.org/10.1007/s40996-018-0192-2

[11] Kaveh, A., Rahami, H., Mehanpour, H. "Static and modal analyses of structures with different repeated patterns", Advances in Engineering Software, 51, pp. 1-9, 2012. https://doi.org/10.1016/j.advengsoft.2012.04.007 or new-Block Circulant Matrices, by using the proposed method, the inverse of the entire structural matrices can be obtained. To maintain the initial properties of the structural matrices, the influence of added parts on the structures is considered. In order to show the accuracy and efficiency of the proposed method, two examples are presented. According to the results, using the properties of new-Block and Block Circulant Matrices can simplify the structural analysis and decrease the computational cost.

[12] Shojaei, I., Kaveh, A., Rahami, H., Shirazi, R., Bazrgari, B "Analysis and reanalysis of mechanical systems: concept of global near-regularity", Acta Mechanica, 228(4), pp. 1445-1456, 2017. https://doi.org/10.1007/s00707-016-1778-z

[13] Kaveh, A., Nikbakht, M., Rahami, H. "Improved group theoretic method using graph products for the analysis of symmetric-regular structures", Acta Mechanica, 210(3-4), pp. 265-289, 2010. https://doi.org/10.1007/s00707-009-0204-1

[14] Kaveh, A., Rahami, H., Nikbakht, M. "Vibration analysis of regular structures by graph products: Cable networks", Computers and Structures, 88(9-10), pp. 588-601, 2010. https://doi.org/10.1016/j.compstruc.2010.02.001

[15] Kaveh, A., Fazli, H. "Approximate eigensolution of locally modified regular structures using a substructuring technique", Computers and Structures, 89(5-6), pp. 529-537, 2011. https://doi.org/10.1016/j.compstruc.2010.12.013

[16] Kaveh, A., Fazli, H. "Canonical Forms for Symmetric and Regular Structures", Journal of Mathematical Modelling and Algorithms, 11(2), pp. 119-157, 2012 https://doi.org/10.1007/s10852-011-9170-4

[17] Kaveh, A., Fazli, H. "Free vibration analysis of locally modified regular structures using shifted inverse iteration method", Computers and Structures, 108-109, pp. 75-82, 2012. https://doi.org/10.1016/j.compstruc.2012.02.013

[18] Kaveh, A., Shojaei, I., Rahami, H. "New developments in the optimal analysis of regular and near-regular structures: decomposition, graph products, force method", Acta Mechanica, 226(3), pp. 665$681,2015$. https://doi.org/10.1007/s00707-014-1194-1

[19] Koohestani, K., Kaveh, A. "Efficient buckling and free vibration analysis of cyclically repeated space truss structures", Finite Elements in Analysis and Design, 46(10), pp. 943-948, 2010. https://doi.org/10.1016/j.finel.2010.06.009

[20] Kaveh, A., Shojaei, I. "Advances in swift analysis of structures: Near-regular structures, and optimal analysis and design", Advances in Engineering Software, 90, pp. 119-126, 2015. https://doi.org/10.1016/j.advengsoft.2015.08.004

[21] Logan, D. L. "A first course in the finite element method", 5th ed., Cengage Learning, Boston, MA, USA, 2011.

[22] Szilard, R. "Theories and applications of plate analysis: classical, numerical, and engineering methods", John Wiley \& Sons, Hoboken, NJ, USA, 2004. https://doi.org/10.1002/9780470172872 